PROCEEDINGS OF THE

AMERICAN MATHEMATICAL SOCIETY

Volume 130, Number 7, Pages 2125-2131

S 0002-9939(02)06311-6

Article electronically published on January 17, 2002

\title{
A DIMENSIONAL RESULT FOR RANDOM SELF-SIMILAR SETS
}

\author{
YAN-YAN LIU AND JUN WU
}

(Communicated by Claudia M. Neuhauser)

\begin{abstract}
A very important property of a deterministic self-similar set is that its Hausdorff dimension and upper box-counting dimension coincide. This paper considers the random case. We show that for a random self-similar set, its Hausdorff dimension and upper box-counting dimension are equal a.s.
\end{abstract}

\section{INTRODUCTION}

The notation of self-similarity is of increasing interest in many theoretical and applied settings, such as random processes, turbulence, and dynamical systems. Self-similar sets are usually fractal sets, i.e. sets of non-integer Hausdorff dimension. If a compact set $K$ is the union of sets $K_{1}, K_{2}, \cdots, K_{N}$, which are similar to $K$ in the ratio $r_{i} \leq 1, i=1,2, \cdots, N$, and $K_{i} \cap K_{j}$ is "almost empty" (precisely open set condition), then $\operatorname{dim}_{H} K=\overline{\operatorname{dim}}_{B} K=\alpha$, where $\alpha$ is determined by the following equation:

$$
\sum_{i=1}^{N} r_{i}^{\alpha}=1
$$

This was made precise by Hutchinson in 6]. It is much more difficult to determine the dimension for self-similar sets with overlap. Falconer [3] (see also [4], Chapter 3 ) proved that a self-similar set (overlap or not) has a very nice property, that is $\operatorname{dim}_{H} K=\overline{\operatorname{dim}_{B}} K$.

The above-mentioned notion of deterministic self-similarity is very restrictive and does not apply to many phenomena. Recently there have been some models of random sets possessing the self-similar property. In particular, Falconer [2], Graf [5] and Mauldin and Williams [8] independently investigated random fractal sets by randomizing each step in Hutchinson's construction. Much more recently, Hutchinson and Ruschendorff [7] extended the contraction mapping method to prove various existence and uniqueness properties of random self-similar sets. For other references on this topic, see [1, 9] and the references therein.

Received by the editors July 16, 2000 and, in revised form, February 14, 2001.

2000 Mathematics Subject Classification. Primary 60D05; Secondary 28A78.

Key words and phrases. Random self-similar set, Hausdorff dimension, box-counting dimension.

This research was supported by the Special Funds for Major State Basic Research Projects. 
In this paper, we prove the analogous result to one of [3], that is, there exists a constant $a$ such that a.s.

$$
\operatorname{dim}_{H} K(\omega)=\overline{\operatorname{dim}}_{B} K(\omega)=a,
$$

where $K(\omega)$ is the random self-similar set. The proofs in [3] cannot extend to the random case directly. In the deterministic case, each ball centered in $K$ with radius at most the diameter of $K$, contains a subset of $K$ which is the image of $K$ under a map with Lipschitz constant comparable to the radius of the ball. This is an important point that underlies the argument in [3]. In the random setting although this is not true for individual realization of $K$, it is true in a certain probabilistic sense.

\section{Notations And Definitions}

In this section, we recall the definitions of various dimensions and random selfsimilar sets and give some properties. A more detailed introduction and the proofs of the properties may be found in 1], 4] and [7.

Let $K \subset \mathbf{R}^{\mathbf{d}}$. For any $s \geq 0$, the $s$-dimensional Hausdorff measure of $K$ is given in the usual way by

$$
\mathbf{H}^{s}(K)=\lim _{\delta \rightarrow 0} \inf \left\{\sum_{i}\left|U_{i}\right|^{s}: K \subset \bigcup_{i} U_{i}, 0<\left|U_{i}\right|<\delta\right\},
$$

where $|\cdot|$ denotes the diameter of a set. This leads to the definition of the Hausdorff dimension of $K$ :

$$
\operatorname{dim}_{H} K=\inf \left\{s: \mathbf{H}^{s}(K)<\infty\right\}=\sup \left\{s: \mathbf{H}^{s}(K)>0\right\} .
$$

Many other definitions of dimensions are encountered in the literature. Let $M_{\epsilon}(K)$ be the smallest number of closed balls of radius $\epsilon$ that cover $K$, and let $N_{\epsilon}(K)$ be the maximum number of disjoint closed balls of radius $\epsilon$ with centers in $K$. We define the lower and upper box-counting dimensions by

$$
\begin{aligned}
& \underline{\operatorname{dim}}_{B} K=\liminf _{\epsilon \rightarrow 0} \frac{\log M_{\epsilon}(K)}{-\log \epsilon}, \\
& \overline{\operatorname{dim}}_{B} K=\limsup _{\epsilon \rightarrow 0} \frac{\log M_{\epsilon}(K)}{-\log \epsilon} .
\end{aligned}
$$

In [4, it was shown that

$$
\begin{aligned}
& \underline{\operatorname{dim}}_{B} K=\liminf _{\epsilon \rightarrow 0} \frac{\log N_{\epsilon}(K)}{-\log \epsilon}=\liminf _{n \rightarrow \infty} \frac{\log N_{2^{-n}}(K)}{n \log 2}=\liminf _{n \rightarrow \infty} \frac{\log M_{2^{-n}}(K)}{n \log 2} \\
& \text { 5) } \overline{\operatorname{dim}}_{B} K=\limsup _{\epsilon \rightarrow 0} \frac{\log N_{\epsilon}(K)}{-\log \epsilon}=\limsup _{n \rightarrow \infty} \frac{\log N_{2^{-n}}(K)}{n \log 2}=\limsup _{n \rightarrow \infty} \frac{\log M_{2^{-n}}(K)}{n \log 2}
\end{aligned}
$$

Finally, we can define packing dimension of $K$ by

$$
\operatorname{dim}_{P} K=\inf \left\{\sup \overline{\operatorname{dim}}_{B} A_{i}, K \subset \bigcup_{i} A_{i}\right\} .
$$

It is well known (see [4]) that

$$
\begin{aligned}
& \operatorname{dim}_{H} K \leq \operatorname{dim}_{B} K \leq \overline{\operatorname{dim}}_{B} K \\
& \operatorname{dim}_{H} K \leq \operatorname{dim}_{P} K \leq \overline{\operatorname{dim}}_{B} K
\end{aligned}
$$


Let $J \subset \mathbf{R}^{d}$ be a fixed compact set with $J=\overline{\operatorname{int}(J)}$. We are given a positive integer $N \geq 2$ and a probability measure $\mu$ on $\operatorname{Sim}^{N}$, where $\operatorname{Sim}$ is the space of all similarities of $\mathbf{R}^{d}$ equipped with the usual topology of uniform convergence on compact sets. In this paper, we assume the following:

\section{Assumptions.}

(I) $S_{i}(J) \subset J$ for all $i=1,2, \cdots, N$ and $\mu$-a.s. $\left(S_{1}, S_{2}, \cdots, S_{N}\right)$.

(II) There exist $0<\hat{r}_{1} \leq \hat{r}_{2}<1$ such that $\hat{r}_{1} \leq \operatorname{Lip}\left(S_{i}\right) \leq \hat{r}_{2}$ for all $i=$ $1,2, \cdots, N$ and $\mu$-a.s. $\left(S_{1}, S_{2}, \cdots, S_{N}\right)$.

In the sequel we often make use of symbolic dynamics. Let $\Sigma=\{1,2, \cdots, N\}^{\mathbf{N}}$ be the code space over the indices $1,2, \cdots, N, \Sigma_{n}=\{1,2, \cdots, N\}^{n}$ the space of all sequence of length $n, n \geq 0, \Sigma_{0}=\emptyset$, and $\Sigma_{*}=\bigcup_{n} \Sigma_{n}$. For $\tau \in \Sigma_{n}$, denote by $|\tau|=n$ the length of $\tau$, and by $\tau \mid k$ the truncation of $\tau$ to the first $k$ entries, $k \leq n$. For any $\tau \in \Sigma_{*}, \sigma \in \Sigma_{*} \cup \Sigma$, define $\tau * \sigma=\left(\tau_{1}, \tau_{2}, \cdots, \tau_{|\tau|}, \sigma_{1}, \sigma_{2}, \cdots\right)$. We write $\tau \prec \sigma$ if there exists a $\eta \in \Sigma_{*} \cup \Sigma$ with $\sigma=\tau * \eta$.

Define the space $\Omega=\left(\operatorname{Sim}^{N}\right)^{\Sigma_{*}}$. Let $\Im$ be the product $\sigma$-algebra on $\Omega$. Taking $\mathbf{P}$ the product measure with $\mu$ on each component, we get our primary probability space $(\Omega, \Im, \mathbf{P})$.

For $\omega \in \Omega$ and $\tau \in \Sigma_{*}$ write

$$
\omega(\tau)=\left(S_{\tau * 1}(\omega), S_{\tau * 2}(\omega), \cdots, S_{\tau * N}(\omega)\right)
$$

and $S_{\emptyset}(\omega)=i d$. By $\Im_{k}$ we denote the $\sigma$-algebra generated by all $S_{\tau}$ with $|\tau| \leq k$. For brevity, for any $\tau \in \Sigma_{*}$, write

$$
\begin{aligned}
& \overline{S_{\tau}}=S_{\tau \mid 1} \circ S_{\tau \mid 2} \circ \cdots \circ S_{\tau|| \tau \mid}, \\
& r_{\tau}=\operatorname{Lip}\left(S_{\tau}\right), \\
& \bar{r}_{\tau}=\operatorname{Lip} \bar{S}_{\tau}=r_{\tau \mid 1} r_{\tau \mid 2} \cdots r_{\tau|| \tau \mid}, \\
& J_{\tau}=\bar{S}_{\tau}(J) .
\end{aligned}
$$

Further, let us introduce shift operators $\Delta_{\tau}: \Sigma_{*} \cup \Sigma \rightarrow \Sigma_{*} \cup \Sigma$ by

$$
\Delta_{\tau}(\sigma)=\tau * \sigma,
$$

and write

$$
T_{\tau} \omega=\omega\left(\Delta_{\tau}\right) .
$$

Then we have $S_{\sigma}\left(T_{\tau} \omega\right)=S_{\tau * \sigma}(\omega)$.

Let us define a random mapping $\pi_{\omega}: \Sigma \rightarrow J$ by

$$
\pi_{\omega}(\sigma)=\lim _{n \rightarrow \infty} \bar{S}_{\sigma \mid n}(\omega)\left(x_{0}\right) .
$$

This limit exists for $\mathbf{P}$-a.s. $\omega \in \Omega$ and does not depend on the choice of $x_{0}$. We call a random compact set $K(\omega)=\pi_{\omega}(\Sigma)$ the random self-similar set.

We call a random subset $\Gamma \subset \Sigma_{*}$ a Markov stopping, if:

(1) For each $\sigma \in \Sigma$ and $\omega \in \Omega$, there exists a unique $\tau \in \Gamma(\omega)$ with $\tau \prec \sigma$, and

(2) $\{\omega \in \Omega: \tau \in \Gamma(\omega)\} \in \Im_{|\tau|}$ for all $\tau \in \Sigma_{*}$.

If $\Gamma$ is a Markov stopping, let $\Im_{\Gamma}$ be the sub- $\sigma$-algebra of $\Im$ generated by $\left\{S_{\eta}\right.$ : there is a $\tau \in \Gamma$ with $\eta \prec \tau\}$.

The set $K(\omega)$ fulfills the following invariance (see [1, 7]). 
Theorem 1. For $\mathbf{P}$-a.s. $\omega \in \Omega$, we have:

(1) $K(\omega)=\bigcap_{n=0}^{\infty} \bigcup_{\sigma \in \Sigma_{n}} \bar{S}_{\sigma}(J)$,

(2) $K(\omega)=\bigcup_{\tau \in \Gamma} S_{\tau}(\omega) K\left(T_{\tau} \omega\right)$, where the $K\left(T_{\tau} \omega\right)$ are i.i.d. copies of $K(\omega)$ and independent of $\Im_{\Gamma}$.

\section{MAIN RESUlT AND ITS PROOF}

In this section, we state and prove the main theorem of this paper.

Lemma 1. Suppose the Assumptions are satisfied and let $K(\omega)$ be the random self-similar set. Then there exists a constant a such that $\mathbf{P}$-a.s.

$$
\operatorname{dim}_{H} K(\omega)=a .
$$

Proof. Suppose there exists $b$ such that

$$
0<\mathbf{P}\left\{\omega: \operatorname{dim}_{H} K(\omega)<b\right\}<1 .
$$

By (2) of Theorem 11 $K(\omega)=\bigcup_{i=1}^{N} S_{i}(\omega) K\left(T_{i} \omega\right)$, thus

$$
\operatorname{dim}_{H} K(\omega)=\max _{1 \leq i \leq N} \operatorname{dim}_{H} S_{i}(\omega) K\left(T_{i} \omega\right)=\max _{1 \leq i \leq N} \operatorname{dim}_{H} K\left(T_{i} \omega\right) .
$$

We have

$$
\begin{aligned}
& \mathbf{P}\left\{\omega: \operatorname{dim}_{H} K(\omega)<b\right\} \\
= & \mathbf{P}\left\{\omega: \max _{1 \leq i \leq N} \operatorname{dim} K\left(T_{i} \omega\right)<b\right\} \\
= & \prod_{i=1}^{N} \mathbf{P}\left\{\omega: \operatorname{dim}_{H} K\left(T_{i} \omega\right)<b\right\} \\
= & \left(\mathbf{P}\left\{\omega: \operatorname{dim}_{H} K(\omega)<b\right\}\right)^{N},
\end{aligned}
$$

and this leads to a contradiction.

For any $\sigma \in \Sigma_{*}$ and $\epsilon>0$, let

$$
I_{\epsilon}^{\sigma}(\omega)=\left\{\tau \in \Sigma_{*}:\left|\bar{S}_{\tau}\left(T_{\sigma} \omega\right)(J)\right| \leq \epsilon,\left|\bar{S}_{(\tau|| \tau \mid-1)}\left(T_{\sigma} \omega\right)(J)\right|>\epsilon\right\} .
$$

Define

$$
\begin{aligned}
& D_{\epsilon}^{\sigma}(\omega)=\left\{A \subset I_{\epsilon}^{\sigma}(\omega): \forall \zeta, \eta \in A, \bar{S}_{\zeta}\left(T_{\sigma} \omega\right)(J) \cap \bar{S}_{\eta}\left(T_{\sigma} \omega\right)(J)=\emptyset\right\}, \\
& \bar{N}_{\epsilon}^{(\sigma)}(\omega)=\max _{A \in D_{\epsilon}^{\sigma}(\omega)} \sharp A .
\end{aligned}
$$

Let $A_{\epsilon}^{\sigma}(\omega)$ be a member of $D_{\epsilon}^{\sigma}(\omega)$ which realizes $\bar{N}_{\epsilon}^{(\sigma)}(\omega)$ and write

$$
A_{\epsilon}^{\sigma}(\omega)=\left\{\tau_{\sigma, 1}(\omega), \tau_{\sigma, 2}(\omega), \cdots, \tau_{\sigma, \bar{N}_{\epsilon}^{(\sigma)}(\omega)}(\omega)\right\} .
$$

For simplicity, write $I_{\epsilon}(\omega)=I_{\epsilon}^{\emptyset}(\omega), D_{\epsilon}(\omega)=D_{\epsilon}^{\emptyset}(\omega), \bar{N}_{\epsilon}(\omega)=\bar{N}_{\epsilon}^{(\emptyset)}(\omega), A_{\epsilon}(\omega)=$ $A_{\epsilon}^{\emptyset}(\omega)$.

Lemma 2. Let the Assumptions be satisfied. There exists a constant $c>0$ such that $\mathbf{P}$-a.s. for any $\epsilon>0$,

$$
\bar{N}_{\epsilon}(\omega) \geq N_{\epsilon}(K(\omega)), \quad \bar{N}_{\epsilon}(\omega) \leq c M_{\epsilon}(K(\omega)) .
$$


Proof. Let $B\left(x_{1}, \epsilon\right), B\left(x_{2}, \epsilon\right), \cdots, B\left(x_{N_{\epsilon}(K(\omega))}, \epsilon\right)$ be $N_{\epsilon}(K(\omega))$ disjoint closed balls of radius $\epsilon$ and center in $K(\omega)$. For any $1 \leq i \leq N_{\epsilon}(K(\omega))$, by Theorem $\square$ (1), there exists $\mathbf{i} \in \Sigma$ such that

$$
\bigcap_{n=1}^{\infty} \bar{S}_{(\mathbf{i} \mid n)}(\omega)(J)=x_{i}
$$

Choose $n_{0}$ such that

$$
\bar{S}_{\left(\mathbf{i} \mid n_{0}\right)}(\omega)(J) \subset B\left(x_{i}, \epsilon\right), \quad \bar{S}_{\left(\mathbf{i} \mid n_{0}-1\right)}(\omega)(J) \backslash B\left(x_{i}, \epsilon\right) \neq \emptyset .
$$

Then we have $\left|\bar{S}_{\left(\mathbf{i} \mid n_{0}-1\right)}(\omega)(J)\right|>\epsilon$.

Now we consider two cases:

(i) If $\left|\bar{S}_{\left(\mathbf{i} \mid n_{0}\right)}(\omega)(J)\right| \leq \epsilon$, choose $\tau^{(i)}=\left(\mathbf{i} \mid n_{0}\right)$.

(ii) If $\left|\bar{S}_{\left(\mathbf{i} \mid n_{0}\right)}(\omega)(J)\right|>\epsilon$, then there exists $l \geq 1$ such that

$$
\left|\bar{S}_{\left(\mathbf{i} \mid n_{0}+l\right)}(\omega)(J)\right| \leq \epsilon, \quad\left|\bar{S}_{\left(\mathbf{i} \mid n_{0}+l-1\right)}(\omega)(J)\right|>\epsilon,
$$

choose $\tau^{(i)}=\left(\mathbf{i} \mid n_{0}+l\right)$. Therefore we have

$$
\bar{N}_{\epsilon}(\omega) \geq N_{\epsilon}(K(\omega)) .
$$

Now we prove the second inequality.

Let $\bar{S}_{\tau^{(i)}}(\omega)(J), i=1,2, \cdots, \bar{N}_{\epsilon}(\omega)$, be $\bar{N}_{\epsilon}(\omega)$ disjoint sets satisfying for any $1 \leq i \leq \bar{N}_{\epsilon}(\omega)$,

$$
\left|\bar{S}_{\tau^{(i)}}(\omega)(J)\right| \leq \epsilon, \quad\left|\bar{S}_{\left(\tau^{(i)}|| \tau^{(i)} \mid-1\right)}(\omega)(J)\right|>\epsilon .
$$

Since $\operatorname{int}(J) \neq \emptyset$, there exist $x \in J$ and $c_{1}>0$ such that $B\left(x, c_{1}\right) \subset J$; then $\bar{S}_{\tau^{(i)}}(\omega)(J)$ contains a closed ball with radius $c_{1} \cdot \bar{r}_{\tau^{(i)}}$.

By Assumption (II),

$$
c_{1} \cdot \bar{r}_{\tau^{(i)}} \geq \frac{\hat{r}_{1}}{|J|} \cdot c_{1} \cdot \bar{r}_{\tau^{(i)}|| \tau^{(i)} \mid-1}|J| \geq \frac{\hat{r}_{1}}{|J|} \cdot c_{1} \cdot \epsilon .
$$

Let $B\left(x_{1}, \epsilon\right), B\left(x_{2}, \epsilon\right), \cdots, B\left(x_{M_{\epsilon}(K(\omega))}, \epsilon\right)$ be $M_{\epsilon}(K(\omega))$ closed balls of radius $\epsilon$ such that $\bigcup_{i=1}^{M_{\epsilon}(K(\omega))} B\left(x_{i}, \epsilon\right) \supset K(\omega)$. For any $1 \leq i \leq M_{\epsilon}(K(\omega))$, define

$$
B_{i}(\omega)=\left\{j: 1 \leq j \leq \bar{N}_{\epsilon}(\omega), \bar{S}_{\tau^{(j)}}(\omega)(J) \cap B\left(x_{i}, \epsilon\right) \neq \emptyset\right\} .
$$

Then for any $j \in B_{i}(\omega), \bar{S}_{\tau^{(j)}}(\omega)(J) \subset B\left(x_{i}, 2 \epsilon\right)$. By volume estimating, we have

$$
\lambda_{d}\left(B\left(x_{i}, 2 \epsilon\right)\right) \geq \sharp B_{i}(\omega) \cdot \lambda_{d}\left(B\left(0, \frac{\hat{r}_{1}}{|J|} \cdot c_{1} \cdot \epsilon\right)\right),
$$

where $\lambda_{d}$ is the $d$-dimensional Lebesgue measure. That is,

$$
\sharp B_{i}(\omega) \leq \frac{(2 \epsilon)^{d}}{\left(\frac{\hat{r}_{1}}{|J|}\right)^{d} \cdot c_{1}^{d} \cdot(\epsilon)^{d}}=\frac{2^{d} \cdot|J|^{d}}{\hat{r}_{1}^{d} \cdot c_{1}^{d}}:=c .
$$

Thus,

$$
M_{\epsilon}(K(\omega)) \geq \frac{1}{c} \bar{N}_{\epsilon}(\omega) .
$$

From Lemma 2 and (5), we immediately get the following corollary. 
Corollary 1. Let the Assumptions be satisfied. Then $\mathbf{P}$-a.s.

$$
\overline{\operatorname{dim}}_{B} K(\omega)=\limsup _{n \rightarrow \infty} \frac{\log \bar{N}_{2^{-n}}(\omega)}{n \log 2} .
$$

Now let us prove the main result of this paper.

Theorem 2. Let the Assumptions be satisfied. Then there exists a constant a such that $\mathbf{P}$-a.s.,

$$
\operatorname{dim}_{H} K(\omega)=\overline{\operatorname{dim}}_{B} K(\omega)=a .
$$

Proof. By Lemma 1 we have that there exists a constant $a$ such that $\operatorname{dim}_{H} K(\omega)=$ a, P-a.s.

For any $n \geq 1$, choose $\epsilon=2^{-n}$, and define a random set $K_{n}(\omega) \subset K(\omega)$ as follows. Let

$$
\begin{aligned}
F_{n, 0}(\omega) & =J \\
F_{n, 1}(\omega) & =\bigcup_{\sigma \in A_{\epsilon}(\omega)} \bar{S}_{\sigma}(\omega)(J) \\
F_{n, 2}(\omega) & =\bigcup_{\sigma \in A_{\epsilon}(\omega)} \bigcup_{\tau \in A_{\epsilon}^{\sigma}(\omega)} \bar{S}_{\sigma * \tau}(\omega)(J), \\
& \cdots \\
F_{n, k}(\omega) & =\bigcup_{\sigma_{1} \in A_{\epsilon}(\omega)} \bigcup_{\sigma_{2} \in A_{\epsilon}^{\sigma_{1}}(\omega)} \cdots \bigcup_{\sigma_{k} \in A_{\epsilon}^{\sigma_{1} * \sigma_{2} * \cdots * \sigma_{k-1}}(\omega)} \bar{S}_{\sigma_{1} * \sigma_{2} * \cdots * \sigma_{k}}(\omega)(J) .
\end{aligned}
$$

Let

$$
K_{n}(\omega)=\bigcap_{k=0}^{\infty} F_{n, k}(\omega)
$$

By the construction of $K(\omega)$, we have that $\left\{F_{n, k}(\omega), k \geq 0\right\}$ is a random recursive construction. This was studied by Mauldin and Williams [8] (see also [2]). On the other hand, by (9) and Theorem 1, it is clear that $K_{n}(\omega) \subset K(\omega), \mathbf{P}$-a.s. By Theorem 1.3 and Theorem 3.6 of [8], we have for $\mathbf{P}$-a.s. $\omega$,

$$
\operatorname{dim}_{H} K_{n}(\omega)=b,
$$

where $b$ satisfies the following equation:

$$
\mathbf{E}\left[\sum_{i=1}^{\bar{N}_{2-n}(\omega)}\left(\operatorname{Lip}\left(\bar{S}_{\tau_{\emptyset, i}(\omega)}(\omega)\right)\right)^{b}\right]=1
$$

Therefore

$$
\mathbf{E}\left[\sum_{i=1}^{\bar{N}_{2-n}(\omega)}\left(\operatorname{Lip}\left(\bar{S}_{\tau_{\emptyset, i}(\omega)}(\omega)\right)\right)^{a}\right] \leq 1
$$

that is,

$$
\mathbf{E} \bar{N}_{2^{-n}}(\omega) \leq \frac{|J|^{a} \cdot 2^{n a}}{\hat{r}^{a}}
$$

For any $\delta>0$,

$$
\sum_{n=1}^{\infty} \mathbf{P}\left\{\omega: \bar{N}_{2^{-n}}(\omega) \geq|J|^{a} \cdot 2^{n(a+\delta)}\right\} \leq \sum_{n=1}^{\infty} \frac{\mathbf{E} \bar{N}_{2^{-n}}(\omega)}{|J|^{a} \cdot 2^{n(a+\delta)}} \leq \frac{1}{{\hat{r_{1}}}^{a}} \cdot \sum_{n=1}^{\infty} 2^{-n \delta}<\infty .
$$


By the Borel-Cantelli Lemma, we have

$$
\mathbf{P}\left\{\omega: \bar{N}_{2^{-n}}(\omega) \geq|J|^{a} \cdot 2^{n(a+\delta)} \text { i.o. }\right\}=0 .
$$

By Corollary 1 and (12), we have for P-a.s. $\omega$,

$$
\overline{\operatorname{dim}}_{B} K(\omega) \leq \limsup _{n \rightarrow \infty} \frac{\log |J|^{a} \cdot 2^{n(a+\delta)}}{n \log 2}=a+\delta .
$$

Since $\delta$ is arbitrary, we have for $\mathbf{P}$-a.s. $\omega$

$$
\operatorname{dim}_{H} K(\omega)=\overline{\operatorname{dim}}_{B} K(\omega)=a,
$$

and we finish the proof of Theorem 2 ,

By (7), (8) and Theorem 2 we immediately have the following corollary.

Corollary 2. Let the Assumptions be satisfied. Then there exists a constant a, such that for $\mathbf{P}$-a.s. $\omega$,

$$
\operatorname{dim}_{H} K(\omega)=\operatorname{dim}_{P} K(\omega)=\underline{\operatorname{dim}}_{B} K(\omega)=\overline{\operatorname{dim}}_{B} K(\omega)=a .
$$

\section{ACKNowledgement}

The authors thank the referee for his (her) valuable suggestions.

\section{REFERENCES}

[1] M. Arbeiter and N. Patzschke, Random self-similar multifractals, Math. Nachr. 181(1996), 5-42. MR 97j:28016

[2] K. J. Falconer, Random fractals, Math. Proc. Cambridge Phli. Soc. 100(1986), 559-582. MR 88e:28005

[3] K. J. Falconer, Dimensions and measures of quasi self-similar sets, Proc. Amer. Math. Soc. 106(1989), 543-554. MR 90c:58103

[4] K. J. Falconer, Techniques in Fractal Geometry, John Wiley and Sons, Ltd. Chichester, 1997. MR 99f:28013

[5] S. Graf, Statistically self-similar fractals, Probab. Th. Rel. Fields 74(1987), 358-392. MR 88c:60038

[6] J. E. Hutschinson, Fractal and self-similarity, Indiana Univ. Math. J. 30(1981), 713-747. MR 82h:49026

[7] J. E. Hutschinson and L. Ruschendorff, Random fractal measures via the contraction method, Indiana Univ. Math. J. 47(1998), 471-487. MR 99j:60019

[8] R. D. Mauldin and S. C. Williams, Random recursive constuctions: asymptotic geometric and topological properties, Tran. Amer. Math. Soc. 295(1986), 325-346. MR 87j:60027

[9] N. Patzschke, The strong open set condition in the random case, Proc. Amer. Math. Soc. 125(1997), 2119-2125. MR 97i:28005

Department of Mathematics and Nonlinear Science Center, Wuhan University, Wuhan, Hubei, 430072, People's Republic of China

E-mail address: lisa-yy@263.net

Department of Mathematics and Nonlinear Science Center, Wuhan University, Wuhan, Hubei, 430072, People's Republic of China

E-mail address: wujunyu@public.wh.hb.cn 